\title{
PRIMER REGISTRO DE CORAMBE OBSCURA \\ (NUDIBRANCHIA: CORAMBIDAE) PARA LA COSTA ATLÁNTICA MEXICANA
}

\section{First record of Corambe obscura (Nudibranchia: Corambidae) for the Mexican Atlantic coast}

\author{
Vicencio de la Cruz-Francisco*la, Coral Kaztenny López Torres ${ }^{1 b}$, \\ Gabriel Omar Ramos Téllez ${ }^{1 \mathrm{c}}$ y Jimmy Argüelles-Jiménez ${ }^{2}$
}

\begin{abstract}
${ }^{1}$ Facultad de Ciencias Biológicas y Agropecuarias, Campus Tuxpan, Universidad Veracruzana. Carr. Tuxpan-Tampico km 7.5, Col. Universitaria CP. 92860, Tuxpan, Veracruz, México. *Para correspondencia: vicenciodelacruz@gmail.com. la (D) orcid.org/0000-0001-8339-6730; ${ }^{1 b}$ Dorcid.org/0000-0002-8829-8424; ${ }^{1 c}$ D orcid.org/0000-0001-8283-5797; ${ }^{2}$ Instituto de Ciencias Marinas y Pesquerías, Universidad Veracruzana, Hidalgo 617, Col. Río Jamapa, C.P. 94290, Boca del Río, Veracruz, México. (D) orcid.org/0000-0002-1968-2692.
\end{abstract}

\section{RESUMEN}

Se registra por primera vez la presencia del nudibranquio Corambe obscura para una laguna costera de México. El espécimen se encontró en la laguna de Tampamachoco, Veracruz, sobre colonias de briozoos incrustados en raíces sumergidas de mangle rojo. El nudibranquio presentó un tamaño de $5.6 \mathrm{~mm}$ de longitud y $3 \mathrm{~mm}$ de ancho; es de color amarillo con manchas marrones en el notum y carece de muesca en el notum posterior. El presente registro aumenta el ámbito de distribución original de C. obscura hacia el suroeste del golfo de México.

Palabras clave: nudibranquio, briozoarios, sistema lagunar, mangle rojo, Golfo de México.

\section{ABSTRACT}

The presence of the Corambe obscura nudibranch is recorded for the first time for a coastal lagoon in Mexico. The specimen was found in the lagoon of Tampamachoco, Veracruz, on colonies of bryozoans embedded in red mangrove submerged roots. The nudibranch has a size of $5.6 \mathrm{~mm}$ long and $3 \mathrm{~mm}$ wide; it is yellow with brown spots in the notum and lacks a notch in the posterior notum. This record increases the original distribution range of C. obscura to the southwest of the Gulf of Mexico.

Keywords: nudibranch, bryozarians, lagoon system, red mangrove, Gulf of Mexico.

La familia Corambidae está representada por tres especies del género Loy y por 10 especies del género Corambe (Caballer y Ortea, 2018). Estos nudibranquios son ovalados, aplanados, pequeños y poseen una cutícula notal. Por sus hábitos crípticos se encuentran estrechamente ligados a colonias de briozoarios, de las que se alimentan habitualmente (Cunha et al., 2017; Caballer y Ortea, 2018). En México, el género Corambe ha sido mencionado principalmente para el litoral del Pacifico y está representado por la especie Corambe pacifica MacFarland \& O’Donoghue, 1929 (Flores-Rodríguez et al., 2017). En cambio, de las seis especies de Corambe que tienen distribución en el Atlántico (Martynov y Schrödl, 2011; Caballer y Ortea, 2018), ninguna se ha registrado para las costas mexicanas del golfo y del Caribe mexicano. 
En diciembre de 2018, se recolectó un espécimen de nudibranquio proveniente de la laguna de Tampamachoco (Fig. 1). Este ecosistema lagunar se encuentra en Veracruz, México, entre las coordenadas $21^{\circ} 02^{\prime} 10^{\prime}$ N, $97^{\circ} 22^{\prime} 50^{\prime}$ ' W y $20^{\circ} 58^{\prime} 40^{\prime}$ N, $97^{\circ} 20^{\prime} 29^{\prime}$ W (Fig. 1). Es una laguna somera de elevada turbidez y transparencia media de $0.30 \mathrm{~m}$, presenta una profundidad promedio de $1 \mathrm{~m}$ (Contreras, 1983), las temperaturas superficiales son de $25-30{ }^{\circ} \mathrm{C}$ y la salinidad fluctúa entre 20 y 40 UPS, por lo que es de características polihalina-euhalina (Lara-Domínguez et al., 2011). En primavera, la salinidad se incrementa y disminuye en otoño, mientras la temperatura es mayor en verano y disminuye en invierno (López-Ortega et al., 2012).

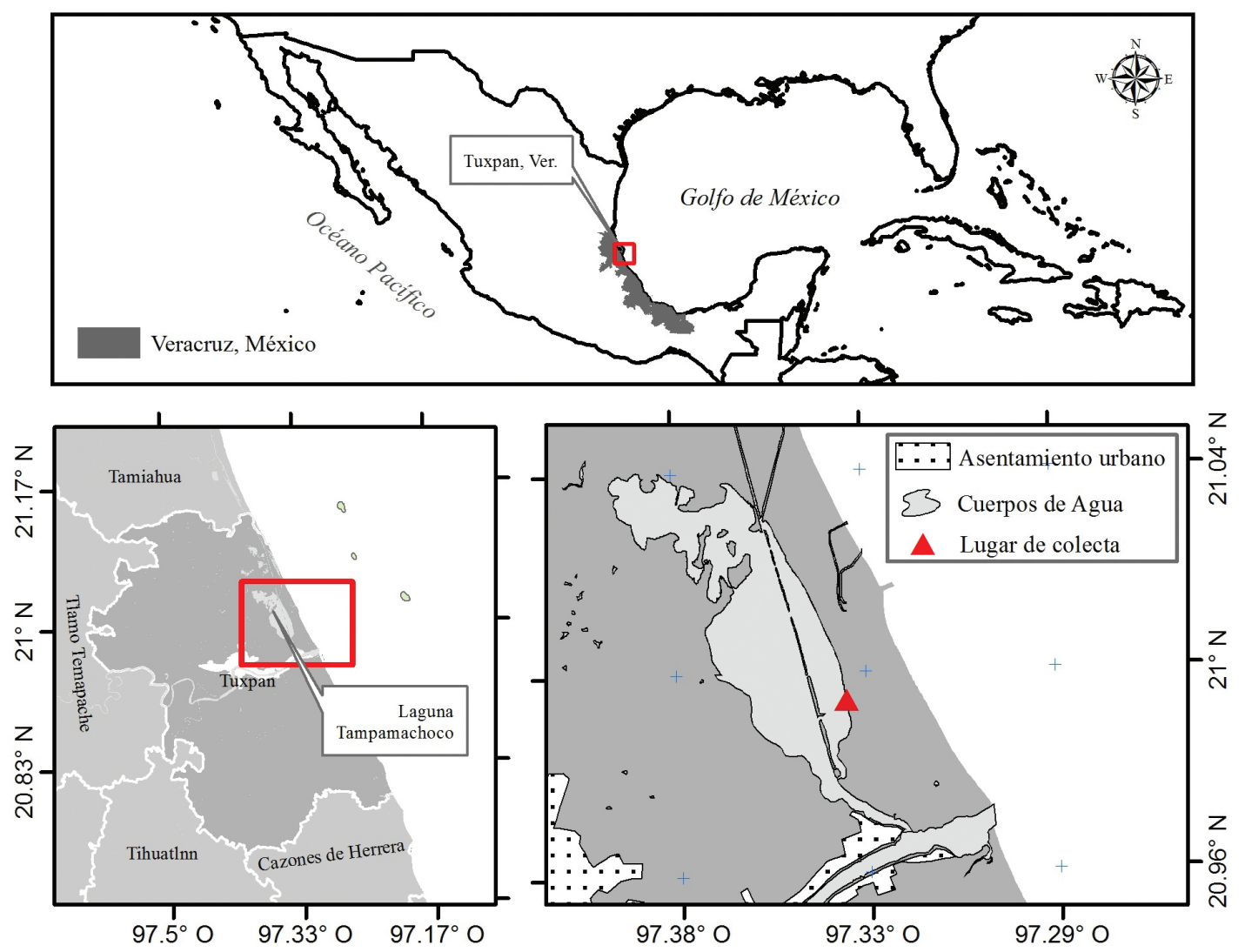

Figura 1. Localización geográfica de la laguna de Tampamachoco y el lugar de colecta de Corambe obscura.

Este sistema lagunar tiene un área de $15 \mathrm{~km}^{2}$, mide aproximadamente $10.6 \mathrm{~km}$ de largo y $2.7 \mathrm{~km}$ de ancho y se encuentra separada del mar por una barrera arenosa conocida como Barra Galindo (Reguero et al., 1991).

El espécimen fue encontrado en raíces sumergidas del mangle rojo (Rhizophora mangle L.) a una profundidad de $0.5 \mathrm{~m}$. La muestra biológica se desprendió manualmente y se conservó en alcohol al $70 \%$. La identificación taxonómica de la especie se realizó con base en la morfología y patrón de coloración que se detallan en los trabajos de Gomoiu y Skolka (1997) y Martynov y Schrödl (2011). El material biológico se depositó en la colección biológica de la Facultad de Ciencias Biológicas de la Universidad Veracruzana. Se consultó la World Register of Marine Species (WoRMS, 2019) para la nomenclatura y las sinonimias. 


\author{
TAXONOMÍA \\ Clase: Gastropoda Cuvier, 1795 \\ Subclase: Heterobranchia Burmeister, 1837 \\ Orden: Nudibranchia Cuvier, 1817 \\ Familia: Corambidae Bergh, 1871 \\ Género: Corambe Bergh, 1869 \\ Corambe obscura (A. E. Verrill, 1870)
}

Sinonimias. Corambe batava Kerbert, 1886; Corambe sargassicola Bergh, 1871; Corambella baratariae Harry, 1953; Corambella depressa Balch, 1899; Doridella obscura A. E. Verrill, 1870.

Material examinado. UVP-000500, sureste de la laguna (20 59 '39.1" N; $\left.97^{\circ} 20^{\prime} 15.7^{\prime \prime} \mathrm{W}\right)$, Tuxpan, Veracruz, México. 0.5 m de profundidad, col. V. De la Cruz (08/XII/2018).

Descripción morfológica. Cuerpo oval y curvado (Fig. 2), tamaño de $5.6 \mathrm{~mm}$ de longitud y $3 \mathrm{~mm}$ de ancho. Notum (superficie dorsal) de color amarillento con protuberancias bajas de color marrón (Fig. 2, A-B). Rinóforos blancos y alargados, con ápices redondeados (Fig. 2, A-B). Branquias y ano en posición posteroventral (Fig. 2, C-D). Borde posterior del notum entero (Fig. 2, A-B). Tentáculos orales en forma de triángulos y fusionados al margen anterior del cuerpo (Fig. 2D). Margen anterior del pie muscular con hendidura (Fig. 2D).

Tipo de localidad. Laguna de Tampamachoco, Tuxpan, Veracruz, México.

Distribución geográfica. Noreste del golfo de México (Rosenberg et al., 2009); Atlántico norte y Mar Negro (Gomoiu y Skolka, 1997; Martynov y Schrödl, 2011). Constituye el primer registro para México.

Observaciones. De las especies del género Corambe, C. obscura es de las pocas que carecen de muesca en el notum posterior (Martinov y Schrödl, 2011). Otra especie con esta característica es C. carambola, la cual se distribuye al sur del Atlántico (Martynov y Schrödl, 2011; Cunha et al., 2017), sin embargo, C. carambola se distingue de C. obscura por presentar un parche blanco brillante en posición ventral (Cunha et al., 2014) que no es observado en el ejemplar colectado (Figura 2, A-D).

Si bien Corambe obscura se restringe a los ambientes marinos, es tolerante a condiciones salobres (Martynov y Schrödl, 2011), esto explica su presencia en la laguna de Tampamachoco, la cual es de características polihalinas-euhalinas (Lara-Domínguez et al., 2011). En el área de estudio, este nudibranquio se encontró sobre briozoarios, la cual es una de sus principales presas (Martynov y Schrödl, 2011). La coloración amarilla y las manchas marrones que presenta C. obscura en el notum, facilitó su observación de entre las colonias de briozoos (Martynov et al., 2011), no obstante, el pequeño tamaño y el movimiento lento podrían ser factores que dificulten su avistamiento en posteriores estudios, por lo que se sugiere realizar revisiones minuciosas.

Varios trabajos han reportado la riqueza malacológica en la laguna de Tampamachoco (Reguero et al., 1991; Lucas y De la Cruz-Francisco, 2018), sin embargo, no citan la presencia de nudibranquios. Por lo tanto, el hallazgo de C. obscura es importante a nivel local y nacional, dado que constituye el primer registro para México, con distribución para el suroeste del golfo de México. Con esta contribución, sustentamos que la familia Corambidae está presente en el litoral del pacifico mexicano con C. pacifica (Flores-Rodríguez et al., 2017) y en las costas mexicanas del golfo de México con $C$. obscura. 

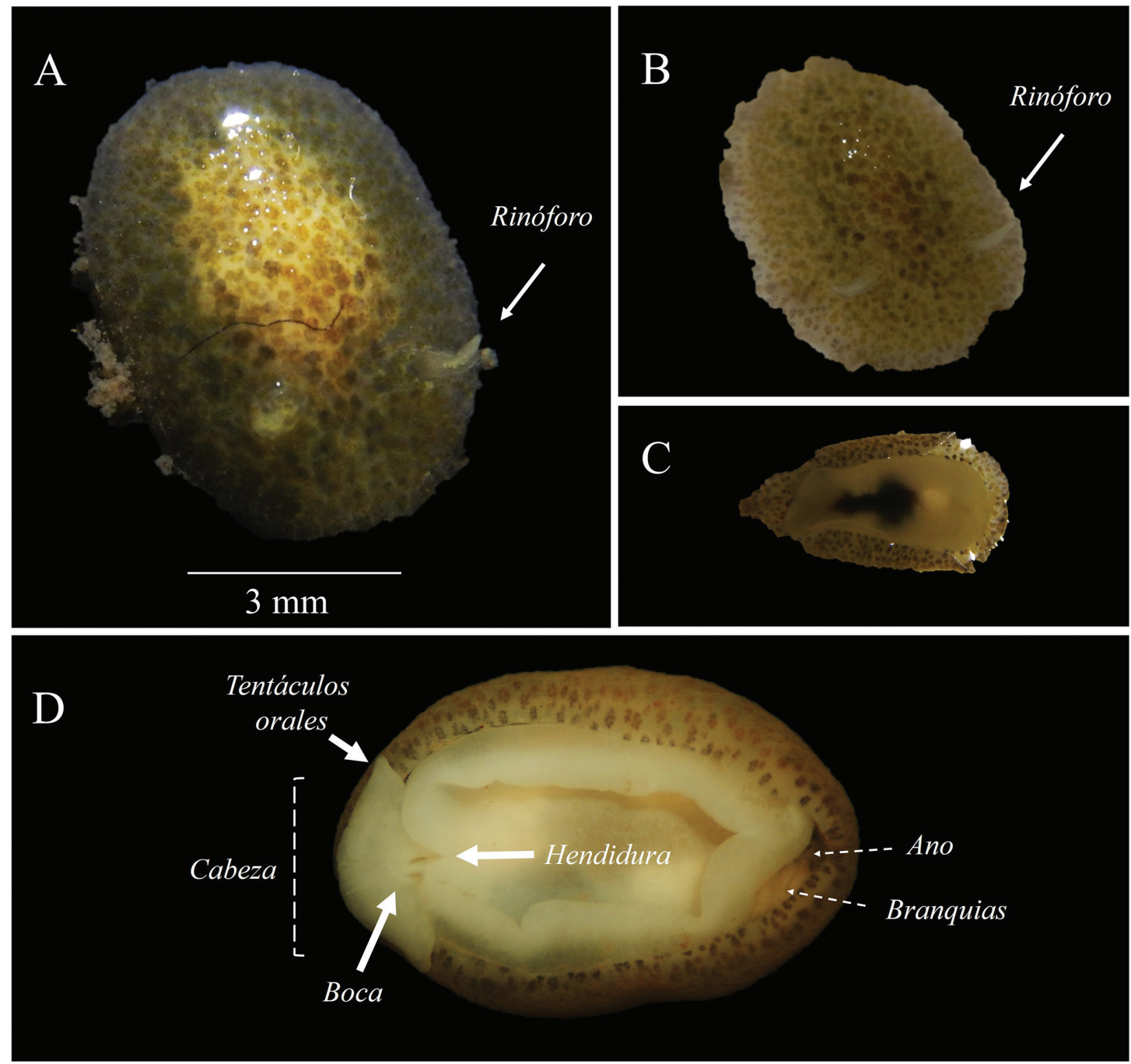

Figura 2. A-B, vista dorsal de Corambe obscura con los rinóforos extendidos. C-D, vista ventral, forma de la cabeza, boca y pie muscular.

\section{LITERATURA CITADA}

Caballer, M. y J. Ortea. 2018. A rare new species of Corambe Bergh, 1869 (Mollusca: Gastropoda: Nudibranchia) from the Caribbean Sea, with a review of the taxonomical history of the Corambidae Bergh, 1871. Marine Biodiversity, 49 (3), 1371-1383. doi.org/10.1007/ s12526-018-0919-0.

Contreras E., F. 1983. Variaciones en la hidrología y concentraciones de nutrientes del área estuarino-lagunar de Tuxpan, Tampamachoco, Veracruz, México. Biótica, 8 (2): 201-213.

Cunha, C. M., L. M. Vieira y A. E. Migotto. 2017. Direct observations of the nudibranch Corambe carabola (Marcus, 1955) preying on the bryozoan Alcyonidium hauffi Marcus, 1939. Marine Biodiversity, 48 (4): 1693-1694. 
Cunha, C. M., L. O. Saad y P. O. V. Lima. 2014. Rediscovery of Brazilian corambids (Gastropoda: Onchidorididae). Marine Biodiversity Record, 7: e14. doi:10.1017/S1755267214000062.

Flores-Rodríguez, P., R. Flores-Garza, S. García-Ibáñez, A. Valdés-González, G. MartínezVásquez, Y. Mora-Marín y E. J. González-Sandoval. 2017. Riqueza, composición de la comunidad y similitud de las especies bentónicas de la Subclase Opistobranchia (Mollusca: Gastropoda) en cinco sitios del litoral de Acapulco, México. Revista de Biología Marina y Oceanografía, 52 (1): 67-80.

Gomoiu, M.-T. y M. Skolka. 1997. A new gastropod - opistobranch at the Romanian Black Sea coast. Geo-Eco-Marina, 2: 201-203.

Lara-Domínguez, A. L., F. Contreras Espinosa, O. Castañeda-López, E. Barba-Macías y M. A. Pérez-Hernández. 2011. Lagunas costeras y estuarios. En: Comisión Nacional para el conocimiento y Uso de la Biodiversidad (Conabio) (Eds.), pp. 301-318. La biodiversidad en Veracruz: Estudio de Estado Veracruz, México. Universidad Veracruzana, Instituto de Ecología, A. C.

López-Ortega, M., G. Pulido-Flores, A. Serrano-Solís, J. C. Gaytán-Oyarzún, W. S. Monks. Sheets y M. A. López-Jiménez. 2012. Evaluación estacional de las variables fisicoquímicas del agua de la Laguna de Tampamachoco, Veracruz, México. Revista Cientifica UDO Agrícola, 12 (3): 713-719.

Lucas M., E. y De la Cruz-Francisco, V. 2018. Macroflora y macrofauna asociada a las raíces de Rhizophora mangle (Rhizophoraceae), en la laguna Tampamachoco, Veracruz, México. Revista Colombiana de Ciencia Animal, 10 (1): 31-42.

Martynov, A. y M. Schrödl. 2011. Phylogeny and evolution of corambid nudibranchs (Mollusca: Gastropoda). Zoological Journal of the Linnean Society, 163: 585-604.

Martynov, A., B. Brenzinger, Y. Hooker y M. Schrödl. 2011. 3D-anatomy of a new tropical peruvian nudibranch gastropod species, Corambe mancorensis, and novel hypotheses on dorid gil ontogeny and evolution. Journal of Molluscan Studies, 77: 129-141.

Reguero, M., A. García-Cubas y G. Zúñiga. 1991. Moluscos de la Laguna de Tampamachoco, Veracruz, México: Sistemática y ecología. Anales del Instituto de Ciencias del Mar y Limnología, 18 (2): 289-328.

Rosenberg, G., F. Moretzsohn y E. F. García. 2009. Gastropoda (Mollusca) of the Gulf of Mexico. En: D. L. Felder \& D. K. Camp (Eds.), pp. 579-699, Gulf of Mexico-Origins, Waters, and Biota. Biodiversity. Texas A \& M Press, College Station, Texas.

WoRMS Editorial Board 2019. World Register of Marine Species. Disponible en http://www. marinespecies.org. Accesado: 28-08-2019. doi:10.14284/170.

[Recibido: 17 de junio, 2019. Aceptado para publicación: 27 de agosto, 2019] 